\title{
Correction to: Point estimate and reference normality interval of MRI-derived myocardial extracellular volume in healthy subjects: a systematic review and meta-analysis
}

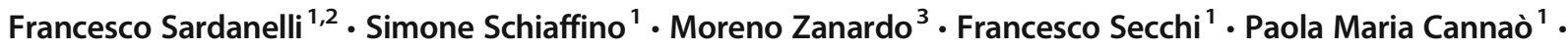 \\ Federico Ambrogi ${ }^{4} \cdot$ Giovanni Di Leo $^{1}$
}

Published online: 5 July 2019

(C) European Society of Radiology 2019

\section{Correction to: European Radiology https://doi.org/10.1007/s00330-019-06185-w}

The original version of this article, published on 02 May 2019, unfortunately contained a mistake. The following correction has therefore been made in the original: The presentation of Fig. 2 was incorrect. The corrected figure is given below.

Publisher's note Springer Nature remains neutral with regard to jurisdictional claims in published maps and institutional affiliations.

The online version of the original article can be found at https://oi.org/ 10.1007/s00330-019-06185-w

Moreno Zanardo

moreno.zanardo@unimi.it

1 Radiology Unit, IRCCS Policlinico San Donato, via Morandi 30, 20097 San Donato Milanese, Milan, Italy

2 Department of Biomedical Sciences for Health, Università degli Studi di Milano, Via Morandi 30, 20097 San Donato Milanese, Milan, Italy

3 Integrative Biomedical Research, Università degli Studi di Milano, Via Mangiagalli 31, 20133 Milan, Italy

4 Department of Clinical Sciences and Community Health, Università degli Studi di Milano, Via Vanzetti 5, 20133 Milan, Italy 
Fig. 2 Forest plot of the 56

analyzed studies, for a total of 64 independent study parts.

Heterogeneity among studies was very high $\left(I^{2}=92 \%\right)$. The last row shows the pooled extracellular volume obtained using the random effects model

\begin{tabular}{|c|c|c|c|c|}
\hline \multirow[t]{3}{*}{ Model } & \multirow[t]{3}{*}{$\underline{\text { Study name }}$} & \multicolumn{3}{|c|}{ Statistics for each study } \\
\hline & & & Lower & Upper \\
\hline & & Mean & limit & limit \\
\hline & Mordi 2017 & 0,270 & 0,254 & 0,286 \\
\hline & Storz 2017 & 0,246 & 0,242 & 0,250 \\
\hline & Wang 2017 & 0,269 & 0,264 & 0,274 \\
\hline & Youn 2017 & 0,258 & 0,248 & 0,268 \\
\hline & Altabella 2016 & 0,245 & 0,228 & 0,262 \\
\hline & Boentert 2016 & 0,254 & 0,242 & 0,266 \\
\hline & Bulluck 2016 & 0,264 & 0,255 & 0,273 \\
\hline & Hanneman 2016 & 0,270 & 0,251 & 0,289 \\
\hline & Lee 2016 & 0,263 & 0,256 & 0,270 \\
\hline & Luetkens 2016 (1a) & 0,277 & 0,261 & 0,293 \\
\hline & Luetkens 2016 (1b) & 0,253 & 0,241 & 0,265 \\
\hline & Luetkens $2016(2 a)$ & 0,265 & 0,250 & 0,280 \\
\hline & Luetkens 2016 (2b) & 0,261 & 0,249 & 0,273 \\
\hline & Mayr 2016 & 0,250 & 0,243 & 0,257 \\
\hline & Mori 2016 & 0,262 & 0,250 & 0,274 \\
\hline & Olivieri 2016 (a) & 0,252 & 0,235 & 0,269 \\
\hline & Olivieri 2016 (b) & 0,207 & 0,195 & 0,219 \\
\hline & Schmacht 2016 & 0,264 & 0,250 & 0,278 \\
\hline & Soslow 2016 & 0,240 & 0,234 & 0,246 \\
\hline & Weingartner 2016 (a) & 0,260 & 0,249 & 0,271 \\
\hline & Weingartner 2016 (b) & 0,202 & 0,193 & 0,211 \\
\hline & Weingartner 2016 (c) & 0,213 & 0,202 & 0,224 \\
\hline & Zhao 2016 & 0,251 & 0,242 & 0,260 \\
\hline & aus dem Siepen 2015 & 0,230 & 0,222 & 0,238 \\
\hline & Bany persad 2015 & 0,250 & 0,245 & 0,255 \\
\hline & Barison 2015 (1) & 0,280 & 0,255 & 0,305 \\
\hline & Barison 2015 (2) & 0,250 & 0,230 & 0,270 \\
\hline & Edwards 2015 (1) & 0,250 & 0,238 & 0,262 \\
\hline & Edwards 2015 (2) & 0,250 & 0,238 & 0,262 \\
\hline & Ertel 2015 & 0,260 & 0,256 & 0,264 \\
\hline & Hong 2015 & 0,256 & 0,241 & 0,271 \\
\hline & Kuruvilla 2015 & 0,260 & 0,247 & 0,273 \\
\hline & Mehta 2015 & 0,271 & 0,259 & 0,283 \\
\hline & Ntusi 2015 & 0,279 & 0,275 & 0,283 \\
\hline & Singh 2015 & 0,251 & 0,243 & 0,259 \\
\hline & Barison 2014 & 0,260 & 0,249 & 0,271 \\
\hline & Brower 2014 & 0,260 & 0,250 & 0,270 \\
\hline & Chin 2014 & 0,260 & 0,253 & 0,267 \\
\hline & Dabir 2014 (a) & 0,250 & 0,237 & 0,263 \\
\hline & Dabir 2014 (b) & 0,260 & 0,246 & 0,274 \\
\hline & Edwards 2014 & 0,250 & 0,243 & 0,257 \\
\hline & Florian 2014 & 0,240 & 0,230 & 0,250 \\
\hline & Luetkens 2014 & 0,236 & 0,224 & 0,248 \\
\hline & Neilan 2014 & 0,270 & 0,253 & 0,287 \\
\hline & Radunski 2014 & 0,250 & 0,244 & 0,256 \\
\hline & Thuny 2014 & 0,268 & 0,259 & 0,277 \\
\hline & Brooks 2013 & 0,268 & 0,254 & 0,282 \\
\hline & Neilan 2013 (1) & 0,280 & 0,270 & 0,290 \\
\hline & Neilan 2013 (2) & 0,280 & 0,270 & 0,290 \\
\hline & Puntmann 2013 (1) & 0,260 & 0,239 & 0,281 \\
\hline & Puntmann 2013 (2) & 0,270 & 0,266 & 0,274 \\
\hline & Salerno 2013 & 0,285 & 0,272 & 0,298 \\
\hline & Shah 2013 & 0,264 & 0,259 & 0,269 \\
\hline & Thompson 2013 & 0,222 & 0,209 & 0,235 \\
\hline & Fontana 2012 & 0,270 & 0,262 & 0,278 \\
\hline & Kawel 2012 (a) & 0,270 & 0,258 & 0,282 \\
\hline & Kawel 2012 (b) & 0,280 & 0,268 & 0,292 \\
\hline & Kawel 2012 (c) & 0,280 & 0,268 & 0,292 \\
\hline & Kellman 2012 & 0,254 & 0,248 & 0,260 \\
\hline & Mongeon 2012 & 0,240 & 0,227 & 0,253 \\
\hline & Sado 2012 & 0,253 & 0,245 & 0,261 \\
\hline & Ugander 2012 & 0,270 & 0,262 & 0,278 \\
\hline & Broberg 2010 & 0,248 & 0,238 & 0,258 \\
\hline & Jerosch-Herold 2008 & 0,240 & 0,220 & 0,260 \\
\hline ando & & 0,256 & 0,252 & 0,260 \\
\hline
\end{tabular}

Mean and $95 \%$ CI

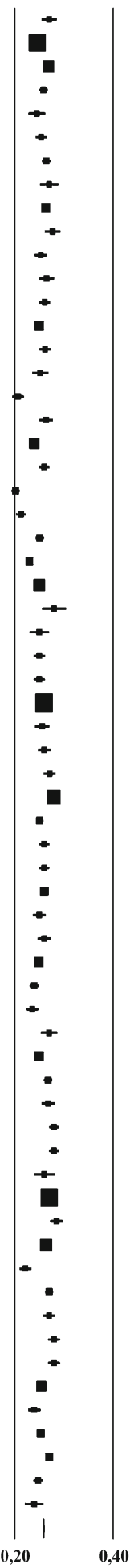

